\title{
RESOLUTION OF THE SIGN AMBIGUITY IN THE DETERMINATION OF THE CYCLOTOMIC NUMBERS OF ORDER 4 AND THE CORRESPONDING JACOBSTHAL SUM
}

\author{
S. A. KATRE and A. R. RAJWADE
}

\section{Introduction.}

In his Werke ([7, pp. 79-87]), Gauss, by a wholly elementary procedure, obtained formulae for cyclotomic numbers of order 4 for a prime $p \equiv 1(\bmod 4)$, in terms of the quadratic partition $p=s_{0}^{2}+t_{0}^{2}, s_{0} \equiv 1$ $(\bmod 4)$, (which fixes $s_{0}$ uniquely and $t_{0}$ upto sign). This result may also be found in the work of Dickson ([5, pp. 400-401]). However, Gauss and Dickson did not resolve the sign ambiguity in $t_{0}$, viz. given a generator $g$ of $F_{p}^{*}$, it is not clear which sign of $t_{0}$ gives correct formulae for the cyclotomic numbers corresponding to $g$. The corresponding result of $M$. Hall ([8, Theorem 3.2]) for $\boldsymbol{F}_{q}\left(q=p^{n} \equiv 1(\bmod 4)\right)$ also has a similar sign ambiguity in the case when $p \equiv 1(\bmod 4)$. (See also Storer $\left[13\right.$, Lemmas $19,19^{\prime}$, p. 48 and p. 51].) The main object of this paper is to resolve this sign ambiguity. Indeed we prove the following:

THEOREM 1 . Let $p$ be an odd prime, $q=p^{n} \equiv 1(\bmod 4), q=1+4 f$. Let $v$ be a generator of $F_{q}^{*}$. If $p \equiv-1(\bmod 4)$, let $s=(-p)^{n / 2}$ and $t=0$. If $p$ $\equiv 1(\bmod 4)$, define $s$ uniquely by $q=s^{2}+t^{2}, p \nmid s, s \equiv 1(\bmod 4)$, and then $t$ uniquely by $v^{(q-1) / 4} \equiv s / t(\bmod p)$. Then the cyclotomic numbers of order 4 for $\boldsymbol{F}_{q}$, corresponding to $v$, are determined unambiguously by the following formulae:

For $f$ even,

$$
\begin{aligned}
& A=(0,0)=\frac{1}{16}(q-11-6 s) \\
& B=(0,1)=(1,0)=(3,3)=\frac{1}{16}(q-3+2 s+4 t) \\
& C=(0,2)=(2,0)=(2,2)=\frac{1}{16}(q-3+2 s) \\
& D=(0,3)=(3,0)=(1,1)=\frac{1}{16}(q-3+2 s-4 t), \\
& E=(1,2)=(2,1)=(1,3)=(3,1)=(2,3)=(3,2)=\frac{1}{16}(q+1-2 s),
\end{aligned}
$$


and for $f$ odd,

$$
\begin{aligned}
& A=(0,0)=(2,0)=(2,2)=\frac{1}{16}(q-7+2 s), \\
& B=(0,1)=(1,3)=(3,2)=\frac{1}{16}(q+1+2 s-4 t), \\
& C=(0,2)=\frac{1}{16}(q+1-6 s), \\
& D=(0,3)=(1,2)=(3,1)=\frac{1}{16}(q+1+2 s+4 t), \\
& E=(1,0)=(1,1)=(2,1)=(2,3)=(3,0)=(3,3)=\frac{1}{16}(q-3-2 s) .
\end{aligned}
$$

(This solves the cyclotomic problem in this case completely.)

Also, for $p \equiv 1(\bmod 4)$, if $s_{0}, t_{0}$ are uniquely determined by $p=s_{0}^{2}+t_{0}^{2}$, $s_{0} \equiv 1(\bmod 4)$, and $v^{(q-1) / 4} \equiv s_{0} / t_{0}(\bmod p)$, then the $s$ and $t$ in the above formulae are given by

$$
s=s_{0}^{n}-\left(\begin{array}{l}
n \\
2
\end{array}\right) s_{0}^{n-2} t_{0}^{2}+\left(\begin{array}{l}
n \\
4
\end{array}\right) s_{0}^{n-4} t_{0}^{4}-\ldots
$$

and

$$
t=t_{0}\left[\left(\begin{array}{l}
n \\
1
\end{array}\right) s_{0}^{n-1}-\left(\begin{array}{l}
n \\
3
\end{array}\right) s_{0}^{n-3} t_{0}^{2}+\ldots\right] .
$$

The above described ambiguity for cyclotomic numbers of order 4 runs parallel to the sign ambiguity in the well known Jacobsthal sum defined by

$$
\Phi_{2}(a)=\Phi_{2}(a ; q)=\sum_{x \in F_{q}}\left(\frac{x\left(x^{2}+a\right)}{q}\right), \quad a \in F_{q}^{*}, \quad\left(q=p^{n} \equiv 1(\bmod 4)\right),
$$

where $(. / q)$ is the Legendre symbol in $\boldsymbol{F}_{q}$. This ambiguity is apparent in the following result of Jacobsthal ( $q=p$ case) (see also [1, pp. 384-385]).

Proposition (Jacobsthal [10], 1907). For $p \equiv 1(\bmod 4), p=s_{0}^{2}+t_{0}^{2}$, $s_{0} \equiv 1(\bmod 4)$, one has,

$$
\Phi_{2}(a ; p)=\begin{aligned}
-2 s_{0}, & \text { if } a \text { is a fourth power }(\bmod p), \\
2 s_{0}, & \text { if } a \text { is a square but not a fourth power }(\bmod p), \\
\pm 2 t_{0}, & \text { if } a \text { is not } a \text { square }(\bmod p) .
\end{aligned}
$$

The ambiguity in the sign of $t_{0}$ in the results of Jacobsthal remained unresolved for quite some time. In 1935, Davenport and Hasse ([4, § 7 II, pp. 176-178]) obtained $\Phi_{2}(a)$ in terms of the two normalized prime factors $\pi, \bar{\pi}$ of $p=\pi \bar{\pi}$ in $\mathrm{Z}[i]$ and the quartic residue symbol. (See the formulation of H. P. F. Swinnerton-Dyer in [3, p. 284] for the case $q=p$. From the formuls for $N_{p}$ there, we get $\Phi_{2}(a ; p)$ by $N_{p}=p+1+\Phi_{2}$ $(-D ; p)$.) The ambiguity in Jacobsthal's result was resolved by $E$. Lehmer $([12$, Theorems 2 and 4$]$ ) in the case when 2 is a quartic nonresidue of $p \equiv 1(\bmod 4)$. 
Recently, Hudson and Williams [9], Evans [6], and Katre (independently) [11], have resolved this sign ambiguity completely in the case $q=p$ by different methods. However for $q=p^{n}(n>1)$ only partial results are known. For $q=p^{2}$, see the results of Berndt and Evans (with sign ambiguities) in [2, Theorems 6.1 and 6.2]. For a general $q$, the result for $\Phi_{2}(1)$ may be found in Storer's book [13, p. 56]. The second aim of this paper is to obtain unambiguous results for $\Phi_{2}\left(a, p^{n}\right), n \geqq 1$. This is achieved in the following

THeOREM 2. Let $q=p^{n} \equiv 1(\bmod 4)$. Let $a \in F_{q}, a \neq 0$. If $p \equiv-1(\bmod 4)$, let $s=(-p)^{n / 2}$ and $t=0$. If $p \equiv 1(\bmod 4)$, define $s$ uniquely by $q=s^{2}+t^{2}$, $p \nmid s, s \equiv 1(\bmod 4)$, and in case $a$ is not a square in $\boldsymbol{F}_{q}$, define $t$ uniquely in terms of a by $a^{(q-1) / 4} \equiv s / t(\bmod p)$. Then $\Phi_{2}(a)$ is unambiguously given by

$$
\Phi_{2}(a)=\begin{aligned}
-2 s & \text { if } a \text { is a fourth power in } \boldsymbol{F}_{q}, \\
2 s & \text { if } a \text { is a square but not a fourth power in } \boldsymbol{F}_{q}, \\
2 t & \text { if } a \text { is not a square in } \boldsymbol{F}_{q} .
\end{aligned}
$$

Also, for $p \equiv 1(\bmod 4), q=p^{n}$, if $s_{0}$ and $t_{0}^{2}$ are uniquely given by $p=s_{0}^{2}$ $+t_{0}^{2}, s_{0} \equiv 1(\bmod 4)$, and in case $a$ is not a square in $F_{q}, t_{0}$ is uniquely given by $a^{(q-1) / 4} \equiv s_{0} / t_{0}(\bmod p)$, then we have the alternative formulation

$$
\begin{aligned}
& -2\left[s_{0}^{n}-\left(\begin{array}{l}
n \\
2
\end{array}\right) s_{0}^{n-2} t_{0}^{2}+\ldots\right] \\
& \Phi_{2}(a)=2\left[s_{0}^{n}-\left(\begin{array}{l}
n \\
2
\end{array}\right) s_{0}^{n-2} t_{0}^{2}+\ldots\right] \quad \begin{array}{l}
\text { if } a \text { is a square but not } a \\
\text { fourth power in } F_{q},
\end{array} \\
& 2 t_{0}\left[\left(\begin{array}{l}
n \\
1
\end{array}\right) s_{0}^{n-1}-\left(\begin{array}{l}
n \\
3
\end{array}\right) s_{0}^{n-3} t_{0}^{2}+\ldots\right] \text { if } a \text { is not a square in } F_{q} \text {. }
\end{aligned}
$$

In section 2, we give the proof of Theorem 1 and in section 3, that of Theorem 2. In section 4 , we give an example.

Remark. For $a, b \neq 0$ in $\boldsymbol{F}_{q}(q \equiv 1(\bmod 4))$, Theorem 1 enables us to find the number $N_{1}$ of solutions of the equation $a x^{4}-b y^{4}=1$ in $F_{q}$, this number being $16(k, h)+N_{0}(a)+N_{0}(-b)$, where for any chosen generator $v$ of $F_{q}^{*}, h \equiv \operatorname{ind}_{v} a(\bmod 4), k \equiv \operatorname{ind}_{v} b(\bmod 4)$, and for $u \in F_{q}^{*}, N_{0}(u)=4$ or 0 according as $u$ is or is not a fourth power in $\boldsymbol{F}_{q}$. Also, Theorem 2 enables us to find the number $N_{2}$ of solutions of $y^{2}=a x^{4}-b, a, b \in F_{q}^{*}$, since 


$$
\begin{aligned}
N_{2} & =q+\sum_{x \in F_{q}}\left(\frac{a x^{4}-b}{q}\right)=q+(a / q) \sum\left(\frac{x^{4}-b / a}{q}\right) \\
& =q+(a / q)\left\{\Phi_{2}(-b / a)-1\right\} .
\end{aligned}
$$

The number of solutions of $y^{2}=x^{3}+a x$ in $F_{q}$ is given by $q+\Phi_{2}(a)$.

\section{The unique determination of cyclotomic numbers of order 4 .}

Let $p$ be an odd prime, $q=p^{n} \equiv 1(\bmod 4), q=1+4 f, v$ be a generator of the cyclic group $\boldsymbol{F}_{q}^{*}, \boldsymbol{F}_{q}$ being the finite field of $q$ elements. Let $\chi$ be the character on $F_{q}$, satisfying $\chi(0)=0, \chi(v)=i$. Then for $h, k$ modulo 4 , the cyclotomic numbers $(h, k)$ and the Jacobi sums $R(h, k)$ of order 4 are defined by

$$
\begin{aligned}
(h, k)= & \text { the number of } v \in \boldsymbol{F}_{q} \text { such that } \operatorname{ind}_{v} v \equiv h(\bmod 4) \text { and } \\
& \operatorname{ind}_{v}(v+1) \equiv k(\bmod 4) \\
R(h, k)= & \sum_{v \in F_{q}} \chi^{h}(v) \chi^{k}(1-v) .
\end{aligned}
$$

(Here $\chi^{0}(0)=0$, unlike on p. 44 in [13].

Note that our $R(h, k)$ is the $J_{\chi}(h, k)$ defined on p. 44 of [13], and so by Lemma 15, p. 44 of [13], it is equal to the $R(h, k)$ defined on p. 43 therein, whenever none of $h, k, h+k$ is divisible by 4 . By Lemma 13 of [13], we have $R(1,1), \overline{R(1,1)}=q$ and by Lemma 14 of $[13], R(1,1)=(-1)^{f}$ $R(2,1)$.

But

$$
\begin{aligned}
R(2,1) & \sum_{v \neq 0,1} \chi^{2}(v) \chi(1-v), \\
= & \sum_{v \neq 0,1}\left(\chi^{2}(v)+1\right) \chi(1-v)-\sum_{v \neq 0,1} \chi(1-v), \\
\equiv & \sum_{v \neq 0,1}\left(\chi^{2}(v)+1\right)+\chi(1)(\bmod (2+2 i)), \\
= & -\chi^{2}(1)+q-2+1, \\
= & q-2 \equiv-1(\bmod (2+2 i)) .
\end{aligned}
$$

This gives $R(1,1) \equiv(-1)^{f+1}(\bmod (2+2 i))$.

We note that if we write $R(1,1)=-s+i$, then $q=s^{2}+t^{2}$, and the congruence condition on $R(1,1)$ is equivalent to saying that $s \equiv 1(\bmod 4)$.

LEMMA 1. If $\alpha, \beta \in Z[i]$ are comprime to $1+i$ and they satisfy $(\alpha)=(\beta)$, and $\alpha \equiv \beta(\bmod (2+2 i))$, then $\alpha=\beta$. 
Proof. By the first condition, $\alpha=\beta \eta$, where $\eta$ is a unit in $Z[i]$, hence a root of unity. Since $\alpha, \beta$ are coprime to $1+i$, the second condition forces that $\eta \equiv 1(\bmod (2+2 i))$. Hence $\eta=1$.

In view of this lemma, $R(1,1)$ is fixed completely if one knows the prime ideal decomposition of $R(1,1)$. We achieve this in what follows:

CASE (i). Let $p \equiv-1(\bmod 4)$. Since $q \equiv 1(\bmod 4)$, we get that $n$ is even and so is $f . p$ itself stays prime in $Z[i]$ and so $R(1,1) \overline{R(1,1)}=q$ gives $(R(1,1))=(p)^{n / 2}$ as ideals. This forces that $R(1,1)=-(-p)^{n / 2}$. We have thus proved

Proposition 1. Let $p \equiv-1(\bmod 4), q=p^{n}, q \equiv 1(\bmod 4)$, then the system of diophantine equations $q=s^{2}+t^{2}, s \equiv 1(\bmod 4)$ has a unique solution viz. $s=(-p)^{n / 2}, t=0$. For this solution, $R(1,1)=-s+i t$.

Case (ii). Let $p \equiv 1(\bmod 4)$. In this case $p$ is the product of two distinct prime ideals in Z[i]. Let $b=v^{(q-1) / 4}$. Then $b \in F_{p}$. By abuse of notation, let $b$ also denote any rational integer $\equiv v^{(q-1) / 4}(\bmod p)$. Then $(b-i)(b+i) \equiv 0$ $(\bmod p)$. One checks at once that there is a unique prime divisor $p$ of $p$ which also divides $b-i$. Then $p=p \bar{p}$. We now have

Lemma 2. Let $J=R(1,1)$. Then $p \mid J$ but $p \nmid \bar{J}$.

Proof. For $k=1,3$, let $\sigma_{k}$ be the automorphism of $\mathrm{Q}(i)$ satisfying $\sigma_{k}(i)$ $=i^{k}$. Thus $J=J^{\sigma_{1}}, \bar{J}=J^{\sigma_{3}}$. Let

$$
S_{k}=\sum_{v \in F_{q}} v^{k(q-1) / 4}(1-v)^{k(q-1) / 4} .
$$

Since $v^{(q-1) / 4} \in F_{p}, S_{k}$ may be considered as an integer modulo $p$. We have,

$$
\begin{aligned}
J^{\sigma_{k}}-S_{k}= & \sum_{v \in F_{q}}\left[\chi^{k}(v) \chi^{k}(1-v)-v^{k(q-1) / 4}(1-v)^{k(q-1) / 4}\right], \\
= & \sum_{v} \chi^{k}(v)\left\{\chi^{k}(1-v)-(1-v)^{k(q-1) / 4}\right\}+ \\
& +\sum_{v}(1-v)^{k(q-1) / 4}\left\{\chi^{k}(v)-v^{k(q-1) / 4}\right\} .
\end{aligned}
$$

Each term in the curly brackets is divisible by $b-i$ modulo $p$. Therefore, $J^{\sigma_{k}} \equiv 0(\bmod p)$ if and only if $S_{k} \equiv 0(\bmod p)$ if and only if $S_{k} \equiv 0(\bmod p)$. Now

$S_{k}=\sum_{v} v^{k(q-1) / 4}(1-v)^{k(q-1) / 4}=\sum_{v} \sum_{j=0}^{k(q-1) / 4}(-1)^{j} v^{k(q-1) / 4+j}\left(\begin{array}{c}k(q-1) / 4 \\ j\end{array}\right)$.

But 


$$
\sum_{v \in F_{q}} v^{j}= \begin{cases}0 & \text { if }(q-1) \chi j \\ q-1 & \text { otherwise }\end{cases}
$$

This gives $S_{1} \equiv 0(\bmod p)$, and

$$
S_{3} \equiv(-1)^{(q-1) / 4}\left(\begin{array}{c}
3(q-1) / 4 \\
(q-1) / 4
\end{array}\right)(\bmod p) .
$$

However for $x=1,2,3$, the exact power of $p$ dividing $(x(q-1) / 4)$ ! is $((q-1) /(p-1)-n) x / 4$. Hence the exact power of $p$ dividing $\left(\begin{array}{c}3(q-1) / 4 \\ (q-1) / 4\end{array}\right)$ is $((q-1) /(p-1)-n) \cdot \frac{1}{4} \cdot(3-1-2)=0$. Thus $S_{3}=0(\bmod p)$. This completes the proof of Lemma 2.

LEMMA 3. $(R(1,1))=\mathfrak{p}^{n}$, as ideals.

Proof. This follows from Lemma 2, noting that $(p)=\mu \bar{p}$ and $R(1,1) \overline{R(1,1)}=q=p^{n}$.

LeMma 4. For $p \equiv 1 \quad(\bmod 4)$, the conditions $R(1,1) \equiv(-1)^{f+1}$ $(\bmod (2+2 i))$, and $(R(1,1))=\mathfrak{p}^{n}$, fix $R(1,1)$ completely.

Proof. This follows from Lemma 1.

REMARK. For $p \equiv 1(\bmod 4)$, the number of solutions $(s, t)$ of the equations $q=s^{2}+t^{2}, s \equiv 1(\bmod 4)$ is equal to the number of ideals a in $Z$ [ $\left.i\right]$ such that $\bar{a} \overline{\mathfrak{a}}=q$. (More precisely, let $\left(s_{0}, t_{0}\right)$ be any given solution of $p=s_{0}^{2}+t_{0}^{2}, s_{0} \equiv 1(\bmod 4)$. Then $(s, t)$ is a solution of $q=s^{2}+t^{2}, s \equiv 1$ $(\bmod 4)$ if and only if $s+i t=\left(s_{0}+i t_{0}\right)^{j}\left(s_{0}-i t_{0}\right)^{n-j}$ for some $\left.0 \leqq j \leqq n.\right)$ This number is $n+1$. We should like to know which of these $n+1$ solutions gives rise to $R(1,1)=-s+i$. For this we first note that of these $n+1$ solutions there are exactly two solutions such that $p \nmid(-s+i t)$, viz. those for which $-s+i t$ has prime ideal factorization $p^{n}$ or $\bar{p}^{n}$. Hence these two solutions correspond to $R(1,1)$ and $\overline{R(1,1)}$. Also, $p \nmid(-s+i t)$ is equivalent to saying that $p \nmid s$. (This follows from $q=s^{2}+t^{2}$.) We have thus proved

Proposition 2. For $p \equiv 1(\bmod 4)$, if $s$ and $t$ satisfy $q=s^{2}+t^{2}, p \nmid s, s \equiv 1$ $(\bmod 4)$, then $-s+i t=R(1,1)$ or $\overline{R(1,1)}$ and conversely.

Compare with Theorem 3.2 in [7] and Lemma 18 of [10]).

The proposition shows that the diophantine conditions $q=s^{2}+t^{2}, p \nmid s$, $s \equiv 1(\bmod 4)$ determine $s$ uniquely and $t$ upto sign. We now determine which $t$ gives $R(1,1)=-s+i t$ with the aid of the following 
LeMma 5. For $p \equiv 1(\bmod 4)$, let $q=s^{2}+t^{2}, p \nmid s$. Then $p \mid(-s+i t)$ if and only if $b \equiv s / t(\bmod p)$.

Proof. The conditions $q=s^{2}+t^{2}, p \nmid s$ imply that $-s+i t$ is the power of a single prime divisor of $p$. Hence $p \mid(-s+i t)$ if and only if $p \mid(b-i)(-s-i t)$, and noting that $b \equiv i(\bmod p)$ if and only if $-s+i t \equiv t(b-s / t)(\bmod p)$, the lemma follows.

Corollary. For $p=s_{0}^{2}+t_{0}^{2}, p=\left(-s_{0}+i t_{0}\right)$ if and only if $b \equiv s_{0} / t_{0}$ $(\bmod p)$.

Since $\mathfrak{p} \mid R(1,1)$ but not $\overline{R(1,1)}$ we have

Proposition 3. Let $p \equiv 1(\bmod 4)$. Let $s, t \in Z$ be uniquely determined by $q=s^{2}+t^{2}, p \nmid s, s \equiv 1(\bmod 4)$, and $v^{(q-1) / 4} \equiv s / t(\bmod p)$. Then $R(1,1)=-s$ + it and conversely.

LemMa 6. Let $p \equiv 1(\bmod 4)$. Then with obvious notation $R(1,1 ; q)$ $=(-1)^{n+1}(R(1,1 ; p))^{n}$, where $R(1,1 ; p)$ corresponds to a primitive root $g$ $\bmod p$ satisfying $g^{(p-1) / 4} \equiv v^{(q-1) / 4}(\bmod p)$.

Proof. Each side has absolute value $\sqrt{q}$, and each side has prime ideal factorization $p^{n}$ where $p$ is the unique prime divisor of $p$ which also divides $v^{(q-1) / 4}-i=g^{(q-1) / 4}-i$. Also, if $q=1+4 f$ and $p=1+4 f_{0}$, then $(-1)^{f}$ $=(-1)^{n f_{0}}$, so each side has the same residue $(-1)^{f+1}(\bmod (2+2 i))$. Hence the result follows by Lemma 1.

Proposition 4. Let $p \equiv 1(\bmod 4)$. Let $s$ and $t$ be as in Proposition 3 and let $s_{0}, t_{0}$ be uniquely determined by $p=s_{0}^{2}+t_{0}^{2}, s_{0} \equiv 1(\bmod 4)$, and $v^{(q-1) / 4}$ $\equiv s_{0} / t_{0}(\bmod p)$. Then

$$
s=s_{0}^{n}-\left(\begin{array}{l}
n \\
2
\end{array}\right) s_{0}^{n-2} t_{0}^{2}+\left(\begin{array}{c}
n \\
4
\end{array}\right) s_{0}^{n-4} t_{0}^{4}-\ldots,
$$

and

$$
t=t_{0}\left[\left(\begin{array}{l}
n \\
1
\end{array}\right) s_{0}^{n-1}-\left(\begin{array}{l}
n \\
3
\end{array}\right) s_{0}^{n-3} t_{0}^{2}+\ldots\right] .
$$

Proof. Under the given conditions, $R(1,1 ; p)=-s_{0}+i t_{0}$ ( $g$ to be chosen as in Lemma 6). Hence by Lemma 6,

$$
-s+i t=(-1)^{n+1}\left(-s_{0}+i t_{0}\right)^{n}=-\left(s_{0}-i t_{0}\right)^{n} .
$$

Thus 


$$
\begin{aligned}
s-i t= & \left(s_{0}-i t_{0}\right)^{n} \\
= & {\left[s_{0}^{n}-\left(\begin{array}{l}
n \\
2
\end{array}\right) s_{0}^{n-2} t_{0}^{2}+\left(\begin{array}{l}
n \\
4
\end{array}\right) s_{0}^{n-4} t_{0}^{4}-\ldots\right]-} \\
& -i t_{0}\left[\left(\begin{array}{l}
n \\
1
\end{array}\right) s_{0}^{n-1}-\left(\begin{array}{l}
n \\
3
\end{array}\right) s_{0}^{n-3} t_{0}^{2}+\left(\begin{array}{l}
n \\
5
\end{array}\right) s_{0}^{n-5} t_{0}^{4}-\ldots\right] .
\end{aligned}
$$

Remark. In view of Proposition 4 , for $p \equiv 1(\bmod 4)$ one now does not require to find the values of $s$ and $t$ in $q=s^{2}+t^{2}, p \nmid s, s \equiv 1(\bmod 4)$, by trial for each $n$ separately; it is sufficient to know the result just for $n=1$. Trials to find both $s$ and $s_{0}$ may also be avoided using the results of Gauss (for $s_{0}$ ) and Storer (for $s$ ) (see Theorem 8, p. 52 of [13]) viz. $2 s_{0}$ is the unique even integer between $-p$ and $p$ which is congruent to $\left(\begin{array}{c}2 f_{0} \\ f_{0}\end{array}\right)(\bmod p)$, where $p=1+4 f_{0}$, and $2 s$ is the unique even integer between $-q$ and $q$ which is congruent to $\left(\begin{array}{c}2 f \\ f\end{array}\right)(\bmod q)$, where $q=p^{n}=1+4 f$.

Lemma 7. Let $q \equiv 1(\bmod 4) .(p \operatorname{may} b e \equiv \pm 1(\bmod 4)$.$) For a given$ generator $v$ of $F_{q}^{*}$, write $R(1,1)=-s+i$. Then the cyclotomic numbers of order 4 for $F_{q}$, related to $v$, are those given in the statement of Theorem 1.

Proof. This result follows from the calculations in the proofs of Lemmas 19 and $19^{\prime}$ on pp. 48-51 of [13]. For $q=p$, the formulae appear in the work of Gauss $([7$, p. 83 and 87$])$ or Dickson ([5, pp. 400-401]).

Proof of Theorem 1. This now follows by combining Propositions 1 , 3, 4 with Lemma 7.

\section{The evaluation of the Jacobsthal sum $\Phi_{2}(a)$ in $F_{q}$.}

The Jacobsthal sum $\Phi_{2}(a)$, for $\boldsymbol{F}_{q}, q \equiv 1(\bmod 4), a \in \boldsymbol{F}_{q}, a \neq 0$, is defined by

$$
\Phi_{2}(a)=\sum_{v \in F_{q}}\left(\frac{v\left(v^{2}+a\right)}{q}\right)
$$

where $(\cdot / q)$ is the Legendre symbol in $\boldsymbol{F}_{\mathbf{q}}$. (The Jacobsthal sum may be defined even if $a=0$ or $q \neq 1(\bmod 4)$, but then it is trivial to evaluate it.) The theory developted in section 2 will enable us to evaluate $\Phi_{2}(a)$ correctly and thus remove the sign ambiguity of Jacobsthal and later authors. To this end we first have

LeMMA 8. $\Phi_{2}(a)=(a / q)[\chi(a) R(1,1)+\overline{\chi(a) R(1,1)}]$. 
Proof. By Theorem 2.7, p. 376 of [2],

$$
\begin{aligned}
\Phi_{2}(a) & =\chi(-1)\left[\chi^{2+1}(a) \chi(4) R(1,1)+\chi^{2+3}(a) \chi(4) R(3,3)\right] \\
& =\chi(-4) \chi^{2}(a)[\chi(a) R(1,1)+\overline{\chi(a) R(1,1)}] .
\end{aligned}
$$

But for $q \equiv 1(\bmod 4),-4$ is a fourth power in $\boldsymbol{F}_{q}$. Also $\chi^{2}(a)=(a / q)$. This proves the lemma.

Proof of Theorem 2. For $q \equiv 1(\bmod 4)$, let $R(1,1 ; q)=-s+i t$, and in case $p \equiv 1(\bmod 4)$, let $R(1,1 ; p)=-s_{0}+i t_{0}$, where $g$ and $v$ are related as in Lemma 6. Then

$$
\begin{aligned}
& s=s_{0}^{n}-\left(\begin{array}{l}
n \\
2
\end{array}\right) s_{0}^{n-2} t_{0}^{2}+\ldots, \quad \text { and } \\
& t=t_{0}\left[\left(\begin{array}{l}
n \\
1
\end{array}\right) s_{0}^{n-1}-\left(\begin{array}{l}
n \\
3
\end{array}\right) s_{0}^{n-3} t_{0}^{2}+\ldots\right] .
\end{aligned}
$$

If $p \equiv-1(\bmod 4), s=(-p)^{n / 2}, t=0$. If $p \equiv 1(\bmod 4), s, s_{0}$ are determined uniquely by $q=s^{2}+t^{2}, p \nmid s, s \equiv 1(\bmod 4)$, and $p=s_{0}^{2}+t_{0}^{2}, s_{0} \equiv 1(\bmod 4)$ respectively. If $a$ is a fourth power in $\boldsymbol{F}_{q}$, then

$$
\begin{aligned}
\Phi_{2}(a) & =-s+i t+(-s-i t) \\
& =-2 s .
\end{aligned}
$$

If $a$ is a square but not a fourth power in $\boldsymbol{F}_{q}$, then

$$
\begin{aligned}
\Phi_{2}(a) & =s-i t+(s+i t) \\
& =2 s .
\end{aligned}
$$

Let now $a$ be a nonsquare in $\boldsymbol{F}_{q}$. Let $v$ be a chosen generator of $\boldsymbol{F}_{q}^{*}$ satisfying

$$
v^{(q-1) / 4} \equiv a^{(q-1) / 4}(\bmod p) .
$$

Then $t, t_{0}$ are uniquely determined by

$$
a^{(q-1) / 4} \equiv \frac{s}{t}=\frac{s_{0}}{t_{0}}(\bmod p) .
$$

Also it follows that for the chosen $v, \chi(a)=i$.

Thus

$$
\begin{aligned}
\Phi_{2}(a) & =-[i(-s+i t)-i(-s-i t) \\
& =2 t .
\end{aligned}
$$

This proves the theorem. 


\section{An example.}

Let $p=5, q=p^{2}=25=1+4 f$. Thus $f$ is even, $q=s^{2}+t^{2}, s \equiv 1(\bmod 4)$ has three solutions viz. $(-3, \pm 4)$ and $(5,0)$. The condition $p \nmid s$ rejects the last solution. Thus $s=-3, t= \pm 4$. Take

$$
\boldsymbol{F}_{q}=\left\{l+m \omega \mid l, m(\bmod 5), \omega^{3}=1, \omega \neq 1\right\} .
$$

For the generator $v=2+\omega$ of $\boldsymbol{F}_{q}^{*}$,

$$
v^{(q-1) / 4}=v^{6} \equiv-2(\bmod 5) .
$$

Hence the condition $v^{(q-1) / 4} \equiv s / t(\bmod p)$ gives $t=4$. Thus using the formulae in Theorem 1 for $f$ even, the cyclotomic numbers $(i, j)$ of order 4 in $\boldsymbol{F}_{q}$ corresponding to $v$ are correctly given by the matrix

\begin{tabular}{cr|cccc}
$i$ & $j$ & 0 & 1 & 2 & 3 \\
\hline 0 & 2 & 2 & 1 & 0 \\
1 & 2 & 0 & 2 & 2 \\
2 & 1 & 2 & 1 & 2 \\
3 & 0 & 2 & 2 & 2
\end{tabular}

which may be verified by direct calculation also.

For $a=-2+\omega, a^{(q-1) / 4} \equiv s / t(\bmod p)$ gives $t=-4$, so $\Phi_{2}(a)=2 t=-8$ by Theorem 2 . This agrees with the result obtained by direct calculation, since it may be checked that for $x \neq 0, x^{3}+a x$ is a square for 8 values of $x$ and a nonsquare for 16 values of $x$, so that $\Phi_{2}(a)=8-16=-8$.

Note that the $s_{0}, t_{0}$ of Theorems 1 and 2 in these cases are respectively 1,2 and $1,-2$, and they yield correct results.

\section{REFERENCES}

1. B. C. Berndt and R. J. Evans, Sums of Gauss, Jacobi, and Jacobsthal, J. Number Theory 11 (1979), 349-398.

2. B. C. Berndt and R. J. Evans, Sums of Gauss, Eisenstein, Jacobi, Jacobsthal, and Brewer, Illinois J. Math. 23 (1979), 374-437.

3. J. W. S. Cassels and A. Fröhlich, Algebraic number theory, (Proc. Conf. Brighton, 1965), p. 284. Academic Press, London, New York, 1967.

4. H. Davenport and H. Hasse, Die Nullstellen der Kongruenzzetafunktionen in gewissen zyklischen Fallen, J. Reine Angew. Math. 172 (1935), 151-182.

5. L. E. Dickson, Cyclotomy, higher congruences, and Waring's problem, Amer. J. Math. 57 (1935), 391-424.

6. R. J. Evans, Determination of Jacobsthal sums, Pacific J. Math., to appear.

7. C. F. Gauss, Theoria Residuorum Biquadraticorum, Werke, vol. 2 (1876), 67-92.

8. M. Hall, Jr., Characters and cyclotomy, (Proc. Symp. Pure Math. 8), pp. 31-43. Amer. Math. Soc., Providence, R.I., 1965. 
9. R. H. Hudson and K. S. Williams, Resolution of ambiguities in the evaluation of cubic and quartic Jacobsthal sums, Pacific J. Math. 99 (1982), 379-386.

10. E. Jacobsthal, Úber die Darstellung der Primzahlen der Form $4 n+1$ als Summe zweier quadrate, J. Reine Angew. Math. 132 (1907), 238-245.

11. S. A. Katre, Jacobsthal sums in terms of quadratic partitions of a prime, (Proc. conference in Number Theory held at Ootacamund, India, 1984). To appear in Lecture Notes series Springer-Verlag.

12. E. Lehmer, On the number of solutions of $u^{k}+D \equiv w^{2}(\bmod p)$, Pacific J. Math. $5(1955)$, 103-118.

13. T. Storer, Cyclotomy and difference sets I, Markham Publ. Co., Chicago, 1967.

CENTRE FOR ADVANCED STUDY IN MATHEMATICS

PANJAB UNIVERSITY

CHANDIGARH, 160014

INDIA 